\title{
ORIGINAL RESEARCH \\ Bailout Stent Deployment during Coil Embolization of Intracranial Aneurysms
}

E. Yoo

D.J. Kim

D.I. Kim

J.-W. Lee

S.H. Suh
BACKGROUND AND PURPOSE: Self-expandable stents are an effective tool for coil embolization of wide-neck intracranial aneurysms. The purpose of this study was to assess the feasibility and results of bailout stent positioning during rescue situations after deployment of $\geq 1$ coil.

MATERIALS AND METHODS: Among 318 aneurysms treated by coil embolization in 267 patients, 16 patients who were treated by bailout stent deployment were retrospectively reviewed. Bailout procedures were performed to relieve potential parent artery compromise caused by the protruded coil loops or to prevent migration of the unstable coil basket. The size/location of the aneurysm, technical feasibility, successful stabilization rate, and procedure-related complications were evaluated.

RESULTS: The locations of the aneurysms were the internal carotid artery $(n=12)$ and basilar artery $(n=4)$. The mean aneurysm size was $8.3 \mathrm{~mm}$ (range, 3.5-19.4 mm) with hemorrhagic presentation in 3 patients. Relief/prevention of parent artery compromise was achieved by molding the encroached loops back into the sac $(n=11)$, scaffolding the aneurysmal neck in cases with an unstable coil basket ( $n=4)$, and sidetacking the migrated loop to the parent vessel wall $(n=1)$. The procedure was technically successful in $87.5 \%(n=14)$. Satisfactory molding or stabilization of the coil was seen in $75 \%(n=12)$. Unsatisfactory molding of the protruded small coil loop was noted in 2 cases of small aneurysms. Acute in-stent thrombosis was successfully managed by thrombolysis $(n=1)$.

CoNCLUSIONS: Bailout self-expandable stent deployment may be a feasible and effective method for relief/prevention of parent artery compromise or coil migration caused by prolapsed or unstable coil loops during embolization of aneurysms.
$\mathbf{R}_{\text {tite }}^{\mathrm{e}}$ ecent technical advances in the detachable coil devices for the treatment of intracranial aneurysms have contributed to the safety of this treatment. However, technical complications of coil embolization of intracranial aneurysms still remain, including aneurysm perforation and rupture, parent artery occlusion, thromboembolism, and unraveling of the coil. One of the causes of the thromboembolic complication is the herniation of the coil loops into the parent artery. Protruded coil loops may increase the risk of parent artery thrombosis or distal artery thromboembolism. ${ }^{1}$

A self-expandable stent is an effective tool for providing a scaffold across the neck for coil embolization of wide-neck intracranial aneurysms. It is usually deployed in the parent artery before the introduction of detachable coils to provide a mechanical barrier for preventing coil prolapse from the wideneck aneurysm. However, due to the self-expansile and relatively easy navigable nature of these stents, they may be deployed after the detachment of coils to reverse or stabilize the unfavorable coil loop or mesh protruding into the parent artery. The purpose of this study was to assess the feasibility and results of bailout stent deployment during rescue situations after detachment of $\geq 1$ coil in the aneurysm.

Received October 16, 2008; accepted after revision December 4.

From the Department of Radiology, Severance Hospital, Yonsei University College of Medicine, Seoul, Korea.

This work was supported by the Korea Research Foundation Grant funded by the Korean Government (KRF-2008-331-E00246).

Please address correspondence to: Dong Joon Kim, MD, Department of Radiology, Yonsei University College of Medicine, 250 Seongsanno, Seodaemoon-gu, Seoul, Korea 120-752; e-mail: djkimmd@yuhs.ac

Indicates open access to non-subscribers at www.ajnr.org

DOI 10.3174/ajnr.A1482

\section{Materials and Methods}

Retrospective review from a data base of 318 aneurysms in 267 patients treated by coil embolization in our institution from January 2004 to April 2008 was performed. Decisions of treatment technique in all aneurysms were reached by consensus of neurosurgeons and neurointerventionists regarding the patient's clinical status and the surgical or coiling feasibility of the aneurysms. Patients with cerebral aneurysms who were treated by bailout stent deployment were selected from our data base. Bailout procedures were performed when the protruded coil was considered to be at high risk of thromboembolism or parent vessel flow compromise such as the following: 1) the loops/mesh of the coil encroached more than half of the parent vessel diameter, 2) a free end of the migrated/stretched coil loop pulsated along the blood stream or attenuated parent/branch vessel flow, or 3) unstable movements of the coil basket were seen during successive coil deployments in wide-neck aneurysms. The aims of the bailout stent procedure were the following: 1) to mold the encroached mesh back into the aneurysm sac; 2) to stabilize the prolapsed/stretched loop by sidetacking it to the wall, thus precluding further migration; or 3) to provide preventive scaffolding in cases with an unstable coil basket during subsequent coiling. The procedure was considered "satisfactory" if the intended aim had been achieved. "Unsatisfactory" bailout was defined as no or minimal molding of the protruded coil loops into the sac. "Failed" deployment was defined as a failure of navigation/deployment of the stent at the intended site.

Endosaccular embolization with detachable coils (Guglielmi detachable coils [GDC], Boston Scientific, Natick, Mass; Trufill detachable coil system, Cordis, Miami Lakes, Fla; MicroPlex, MicroVention, Aliso Viejo, Calif; Axium, ev3, Irvine, Calif) was performed in the standard method. When bailout stent placement was indicated, we carefully selected the distal parent artery with a microguidewire (Agility, Cordis) and advanced the microcatheter over the microguidewire. Then the microguidewire was exchanged for a 0.014 inch exchange wire (Transend floppy $300 \mathrm{~cm}$, Boston Scientific), which was used to 
advance and deploy the Neuroform stent (Boston Scientific). Additional coil embolization was performed after bailout stent placement whenever it was considered safe and feasible.

Patients with wide-neck unruptured aneurysms had been given dual antiplatelet agents (75-mg clopidogrel [Plavix] and 100-mg aspirin) for at least 3 days before the procedure. During the interventional procedure, 2000- to 4000-IU heparin was intravenously injected to achieve an activated clotting time twice that of the baseline. For bailout stent placement in ruptured aneurysms, the patients were loaded with antiplatelets (Plavix 300 600 mg and aspirin $100 \mathrm{mg}$ ) immediately after the procedure. The patients were placed on 75-mg Plavix and/or 100-mg aspirin once a day orally for at least 6 weeks after the procedure. Prolonged mediation with 100-mg aspirin was then performed.

The location and size of the aneurysm, cause and success rate of bailout procedure, the procedure-related complications, and the initial and follow-up angiographic results were evaluated. The grades of aneurysmal occlusion subsequent to the coiling procedure were divided into complete ( $100 \%$ occluded), nearly complete ( $\geq 95 \%$ occluded), and partial ( $<95 \%$ occluded). Post-stent placement and follow-up parent vessel stenosis were measured at the point of maximum stenosis relative to the normal proximal diameter. Isolated loop protrusions that did not seem to have any direct effect on the parent vessel flow-though at risk of thromboembolism-were not included in the measurement of the stenosis. Follow-up stent-induced in-stent stenosis was considered significant if the stenosis rate was $\geq 50 \%$.

On follow-up cerebral angiography or MR angiography (MRA), aneurysmal occlusion was classified as stable, thrombosis, compaction, and regrowth. "Stable occlusion" was defined as no change in the morphology of the coil mesh or the remnant flow within the aneurysm sac. "Thrombosis" was defined as progressive occlusion of the previous contrast-filling spaces within the aneurysm. "Compaction" was considered to be present if any increase in the area of contrast filling within the sac was seen. "Regrowth" was defined as any enlargement of the aneurysm sac compared with the immediate postembolization angiography. Institutional review board approval was obtained with waiver of informed consent.

\section{Results}

\section{Patient Demographics}

Among 318 aneurysms in 267 patients, stents were used in 79 patients for embolization of the intracranial aneurysms. In 16 of the 79 patients, the stents were used for bailout situations. The patients consisted of 14 women and 2 men; the mean age was 60 years (range, $46-74$ years) (Table). Three patients had presented with subarachnoid hemorrhage (SAH) with Hunt and Hess (HH) scale grades of II, III, and V (cases 1, 4, and 9, respectively). One patient was treated for a recurred aneurysm that developed 8 years after initial coil embolization of an unruptured internal carotid posterior communicating artery (IC-PcomA) aneurysm (case 6). The locations of the aneurysms were the cavernous internal carotid artery (ICA, $n=2$ ), paraophthalmic ICA $(n=1)$, superior hypophyseal artery (SHA, $n=6)$, PcomA, $(n=3)$, and tip of basilar artery $(n=$ 4). The aneurysm size ranged from 3.5 to $19.4 \mathrm{~mm}$ (mean, 8.3 $\mathrm{mm})$. All aneurysms were wide neck $(>4 \mathrm{~mm}$ neck or dometo-neck ratio of $<1.5$ ).

\section{GDC Embolization and Stent Deployment}

The bailout procedures were performed for aims of molding of the encroaching coil loops/mesh $(n=11)$, providing a scaffold for the unstable coil basket $(n=4)$, and sidewall tacking of the migrated coil loop $(n=1)$. The stent-deployment procedures were technically successful in 14 of 16 patients $(87.5 \%)$. Bailout stent deployment failed due to the difficulty of selecting the distal acutely angled parent artery with the microguidewire ( $n=1$, case 8 ) and due to the severe tortuosity of the aorta and the parent vessel $(n=1$, case 13). In the latter case, stabilization of coil was successfully achieved with balloon-remodeled coiling after failure of bailout stent placement. Bailout stent placement was performed in 3 patients due to coil loop protrusion that occurred after balloon deflation during balloon remodeling in wide-neck aneurysms (cases 11, 14, and 16). In 1 patient, rescue balloon assistance for management of the protruded coil loop was attempted but was unsuccessful; thus, the stent was used for bailout (case 15).

\section{Result of the Procedures and Follow-UP}

Satisfactory molding or stabilization of the coil loop or mesh was seen in 12 of 16 patients (75\%). Unsatisfactory molding of the protruded coil loops was noted in 2 cases of small aneurysms, sized 3.5 and $3.6 \mathrm{~mm}$. After bailout stent insertion, additional coil embolizations were performed in 5 of 12 patients, with satisfactory stabilization (cases 2, 6, 7, 9, and 14). Complete or near-complete occlusion of the aneurysm sac was achieved in 12 aneurysms. Partial occlusion of the sac was achieved in 4 aneurysms. Significant coil-induced parent vessel stenosis $(66 \%)$ was noted on the immediate postbailout stent angiogram in 1 patient after failed bailout (case 8 ).

In terms of complications, acute thrombosis developed in 1 patient immediately after unsatisfactory bailout stent placement (case 3). The thrombus was successfully lysed with intraarterial infusion of urokinase 150,000 U and abciximab (ReoPro) $13 \mathrm{mg}$. One patient with $\mathrm{SAH}, \mathrm{HH}$ grade $\mathrm{V}$, died 3 days after coil embolization due to the insults inflicted during the initial hemorrhage (case 9).

Follow-up angiography or MRA was available in 11 of 16 patients, ranging from 5 to 21 months (mean, 11 months). The occlusions of the coiled aneurysms were stable in 8 patients, progressive thrombosis in 2 patients, and compaction in 1 patient. One patient with a large cavernous ICA aneurysm presented with mild coil compaction (case 2). Suspicious asymptomatic significant (53\%) stent-induced in-stent stenosis was seen in a patient on 5-month follow-up MRA (case 14). The patient was followed for 7 months without development of any clinically relevant symptoms.

\section{Illustrative Cases}

Case 4. A 68-year-old woman with SAH was admitted with $\mathrm{HH}$ scale grade II and Fisher grade II. Diagnostic cerebral angiography revealed a $7.5 \times 4.5 \mathrm{~mm}$ lobulated saccular aneurysm with a 5.4-mm-wide neck at the right IC-PcomA junction (Fig 1). Because of the wide neck of this aneurysm, coil embolization was initially performed by using the double microcatheter technique. After successful detachment of the first coil, the patient was given heparin 3000 IU intravenously. However, digital substraction angiography (DSA) performed after the detachment of the third coil revealed encroachment 


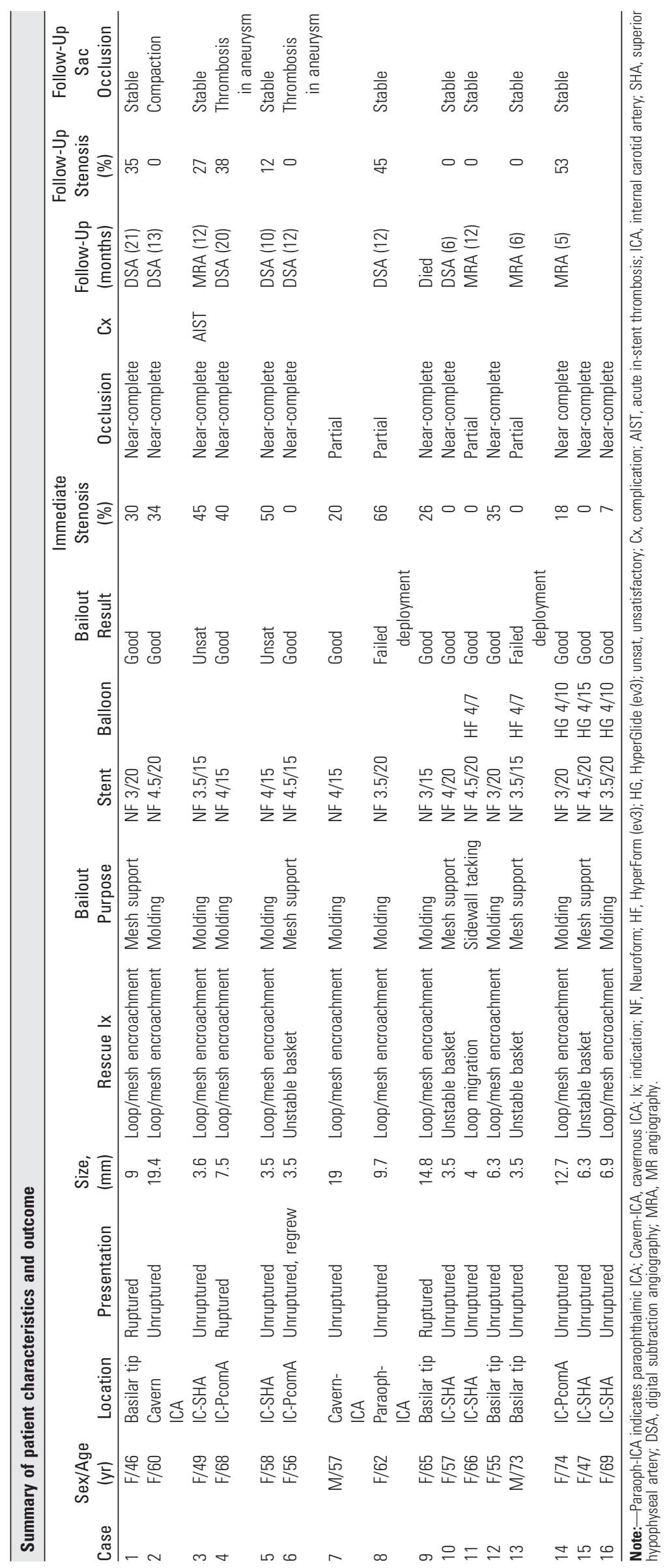



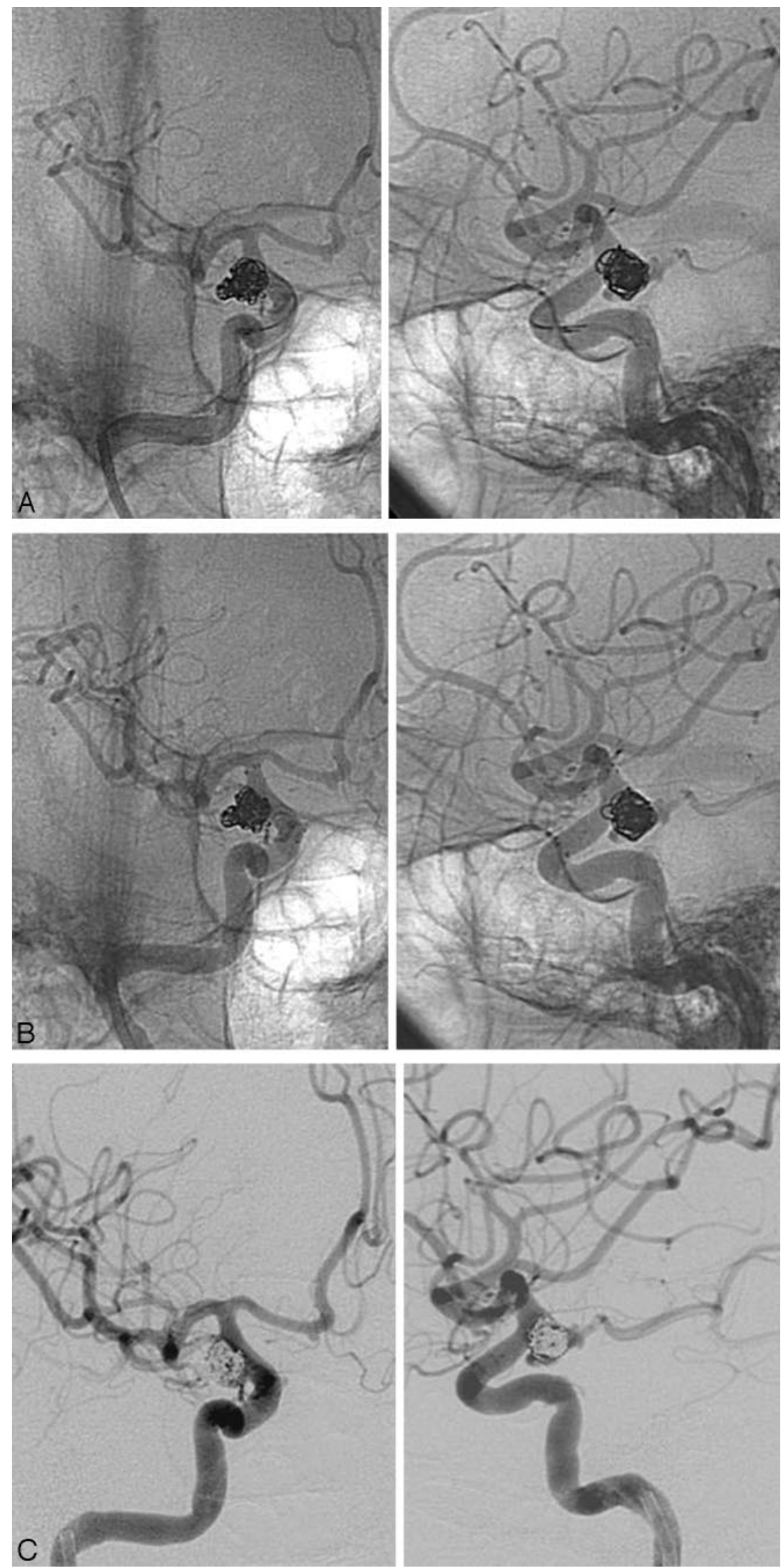

Fig 1. A 68-year-old woman presented with SAH from a right IC-PcomA aneurysm. A double-microcatheter technique was used for coiling of the wide-neck aneurysm. A, Native oblique anteroposterior and lateral views. After detachment of the third coil, encroachment of the coil mesh into the parent artery is noted. $B$, Due to concerns of parent vessel compromise, a $4 \times 15 \mathrm{~mm}$ Neuroform stent was deployed at the site of the protruded coil mesh, achieving successful molding of the protruded coil mesh and restitution of the parent artery lumen. Residual protruding coil loop is seen on the lateral view, which is probably tacked to the sidewall by the stent. C, Subtracted views. Good parent vessel patency is maintained without signs of thrombus formation. 

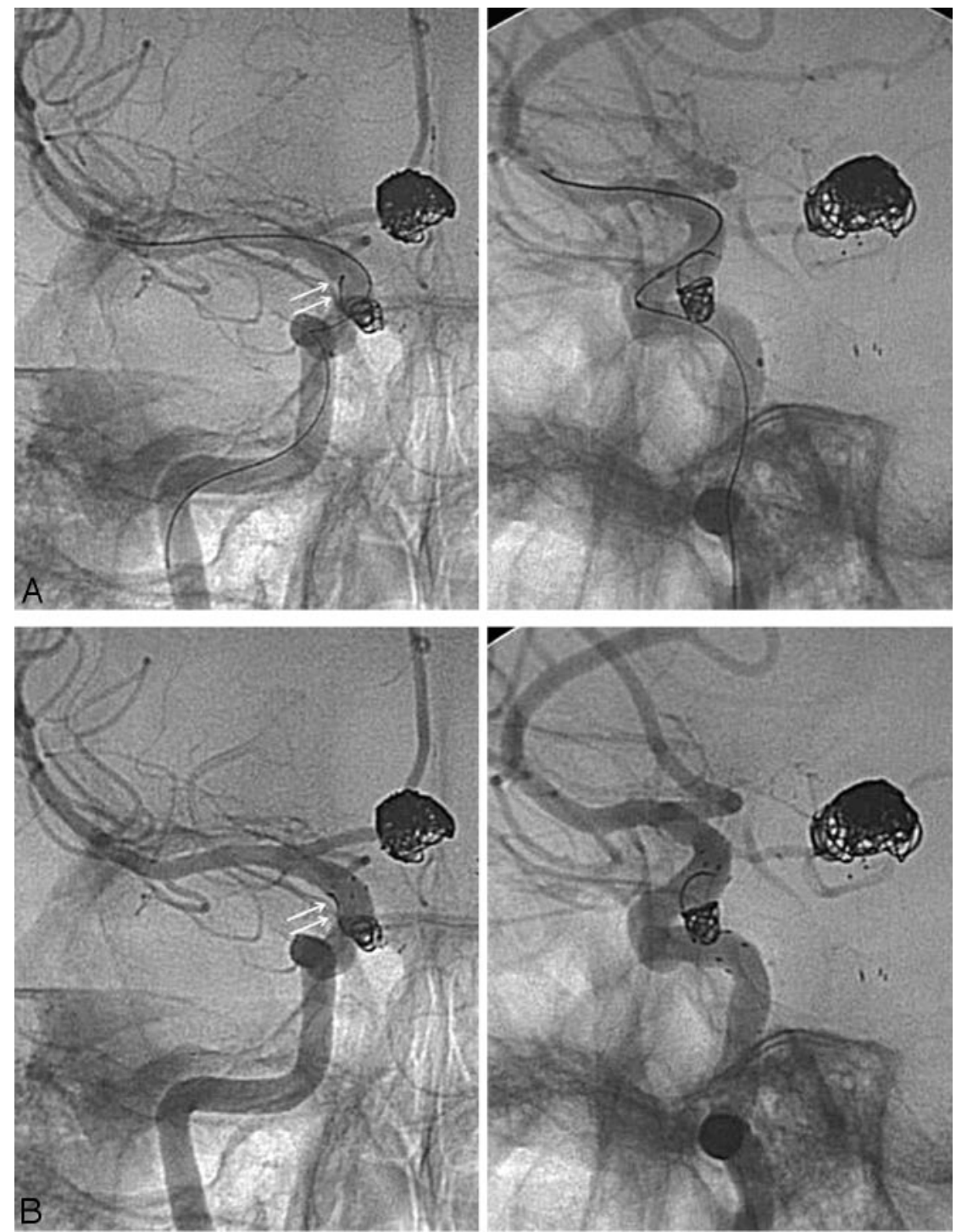

Fig 2. A 66-year-old woman presented with an unruptured right superior hypophyseal aneurysm. $A$, Native anteroposterior/lateral views. During the deployment of the third coil by using balloon remodeling, protrusion and migration of a loop of the previously detached coil was noted (arrows). Pulsatile movement of the migrated distal coil loop was seen. $B, A 4 \times 20$ $\mathrm{mm}$ Neuroform stent is deployed, which successfully sidetacks and stabilizes the migrated loop along the lateral wall of the parent vessel (arrows).

of the coil mesh into the parent artery (Fig $1 A$ ). We decided to use the self-expandable stent for the bailout procedure due to concerns of parent vessel compromise. The distal parent artery was carefully selected by crossing between the protruded coil mesh and the contralateral parent vessel wall. A $4 \times 15 \mathrm{~mm}$ Neuroform stent was navigated and deployed over the microguidewire system with successful molding of the protruded coil mesh and preservation of the parent artery (Fig $1 B$, $-C$ ). The patient was given clopidogrel (Plavix) $75 \mathrm{mg} /$ day immediately after the session, which was continued for 4 months. Aspirin (Astrix) $100 \mathrm{mg} /$ day was given indefinitely. Follow-up angiography 20 months later revealed complete occlusion of the aneurysm without significant in-stent stenosis.

Case 11. A 66-year-old woman was admitted for embolization of an unruptured $4 \times 3.5 \mathrm{~mm}$ right superior hypophyseal aneurysm measuring (Fig 2). She had been treated for a ruptured basilar tip aneurysm 4 months earlier by coil embolization. Due to the relative wide neck of the aneurysm, balloon remodeling was initially attempted. However, during the third coil deployment, a loop of the previously detached coil protruded into the parent artery and partially migrated distally (Fig 2A). Pulsatile movements of the migrated loop were noted. Thus, a $4.5 \times 20 \mathrm{~mm}$ Neuroform stent was deployed to stabilize the protruded coil to the parent artery wall. Poststent angiography revealed the sidetacking of the migrated loop to the lateral aspect of the parent vessel wall with stabilization (Fig 2B). Twelve-month follow-up MRA revealed stable occlusion of the aneurysm.

\section{Discussion}

The results of our case series show that the self-expanding stents used for remodeling of wide-neck aneurysms may be successfully used as a bailout method during the coil embolization procedure. We were able to successfully mold or stabilize the coil loops in $75 \%$ of patients.

One of the most frequent complications associated with 
GDC treatments is the iatrogenic brain ischemia caused by thromboembolic events. This may be caused by thrombus formation from the catheter/guidewire, thrombosis within the aneurysm sac, or herniation of the coils into the parent vessel. ${ }^{2}$ Prevalence of the thromboembolic complication is known to range from $1.0 \%$ to $28 \% .^{2-6}$ A protruded coil loop in the parent artery is one of the significant sources of thrombus formation. ${ }^{7-9}$

The removal of the detached protruding coil loop by using a snare may be the best solution; however, this may be technically difficult and potentially hazardous, with risks of vessel perforation and malpositioning of other coil loops. ${ }^{9}$ It may be better to mechanically mold the protruding coil loops back into the aneurysm sac by inflating the balloon or deploying a stent. Balloon remodeling is a well-known option for treatment of wide-neck aneurysms. ${ }^{10}$ Sugiu et al ${ }^{11}$ were able to successfully replace the protruding coil mass by inflation of the microballoon. However, the balloons used for these procedures are temporary-inflation balloons, and the coils may reprolapse into the parent vessel after deflation. In our series, bailout situations occurred while applying the balloon-remodeling technique in wide-neck aneurysms $(n=3$; cases 11 , 14, and 16). Also, the balloon had been used for bailout with the aim of molding the protruded coil mesh but was unsuccessful ( $n=1$, case 15 ). All 4 of these cases were successfully managed with the deployment of the self-expanding stent for bailout purposes. However, in contrast, there was 1 patient in whom bailout with temporary balloon was successful after failure of stent navigation and deployment (case 13).

Stent-assisted coil embolization is a new tool in the management of intracranial aneurysms and may be particularly useful in the case of the wide-neck or large aneurysm. The stent serves as a mechanical scaffold for the placement of aneurysm coils, prevents coil protrusion into the parent artery, and may allow safer packing of the aneurysm with a denser coil mesh. ${ }^{12-14}$ These mechanical features of the stent may be used to permanently reposition or stabilize the protruded coil loops. Fessler et $\mathrm{al}^{1}$ described a novel method in which a balloon-expandable intracranial stent was used to mold the prolapsed coils by repositioning the loops into the aneurysm sac. However, balloon-expandable stents are generally stiffer than the self-expandable stents that are used for remodeling. The profiles of these balloon-expandable stents are also usually larger with the added balloons. This may be disadvantageous when careful navigation into the distal intracranial vessels beyond the protruded coil loops is necessary, such as in the bailout situation. According to our series, the self-expandable stents were successfully navigated and deployed across the coil mesh in 14 of 16 patients, with satisfactory molding and stabilization of the protruded coil loop or mesh in 12 patients with good follow-up results. Despite the concerns for the insufficient radial force to mold the coils when compared with the balloon-expandable stent, according to our experience, the radial force of these stents $-10 \mathrm{~mm} \mathrm{Hg}$ for the Neuroform stent-was sufficient for such purposes. ${ }^{15}$

However, the successful deployment of the stent across the prolapsed coil loops did not always result in satisfactory molding of the coil loops. These unsuccessful cases were both small aneurysms with diameters of 3.5 and $3.6 \mathrm{~mm}$ and were both located in the convexity of the curved parent vessel (cases 3 and 5). In curved vessels harboring an aneurysm at the convexity, an increased cell opening of the open-cell-designed stent may facilitate such bailout failure. Moreover, these effects of the bailout stent may become more attenuated when the diameter of the coil loops is smaller than the cell size. When a stent with cells of $2 \mathrm{~F}$ in diameter is placed on a convexity, the cells actually open to larger than $2 \mathrm{~F}$, allowing coil prolapse or failing of reposition of the protruded 2- to 3-mmdiameter coils. ${ }^{16}$ Thus, a closed-cell-type stent with smaller cell size may be more effective for bailout of small-diameter coils on the convex surface of the vessel curvature.

All aneurysms treated by bailout stent placement in this study were wide-neck. Fortunately, we did not experience any bailout stent placement situations during treatment of smallneck aneurysms. Although we believe that the stent-assisted technique is effective in the treatment of wide-neck aneurysms, this technique is not always the first choice in our practice due to concerns of potential complications related to the procedure, in-stent stenosis, and long-term antiplatelet medication. If embolization seems feasible with simpler techniques such as complex-type coils, multiple microcatheters, or balloon assistance, these methods are chosen. However, despite the simplicity in using these other techniques, they were not always foolproof and sometimes exposed the patients to bailout situations such as in our experience. Regardless of the neck size or initial choice of embolization technique, bailout stent placement seems to be a feasible option for relief and prevention of parent artery compromise during the embolization procedure.

There are some technical concerns associated with the bailout procedure. Selecting the parent artery distal to the aneurysm with the microguidewire and subsequent advancement of the stent delivery system have possible risks of further disrupting or migrating the previously deployed coils. We were not successful in safely navigating the microguidewire beyond the prolapsed loops in 1 patient because of repeated inadvertent microguidewire selection of the aneurysm sac situated on the acute curvature of the parent vessel. Selection of highly torquable microguidewire with meticulous navigation may be indicated in such situations.

Another concern of using the stents as a bailout procedure is associated with the potential stent-induced thromboembolic complications. With the stent-remodeling technique, patients should be pretreated with antiplatelet drugs, but this is problematic in patients with ruptured aneurysms. It has been shown that the thromboembolism complications rates are high when stents are deployed in the absence of antiplatelet pretreatment. ${ }^{14}$ With respect to the bailout situation in hemorrhagic patients, clopidogrel and aspirin may be administered in loading doses during or shortly after the procedure through a nasogastric tube. ${ }^{7}$ Intra-arterial and intravenous abciximab (ReoPro) administration represents a relatively safe and effective means of achieving pharmacologic lysis of hyperacute thromboemboli identified during endovascular neurointerventional procedures. ${ }^{17}$ The glycoprotein IIb/IIIa (GP IIb/IIIa) inhibitors, which block the final common pathway of platelet aggregation, are suited to prevent the propagation of hyperacute thrombi. According to the previous literature, the 
use of GP IIb/IIIa inhibitors for the management of intraprocedural thromboembolic events has resulted in complete or partial resolution of the thrombus in 33 of 34 cases. ${ }^{17-19}$ Despite the small number of cases, we were able to avoid stentinduced thromboembolic complications by antiplatelet loading immediately after the procedure in nonpremedicated patients. A single patient had developed thrombosis at the site of the protruded coil tip after stent placement (case 3). Bailout stent placement was unsatisfactory in molding the coil loop in this patient. The thrombus was successfully managed by intra-arterial infusion of urokinase 150,000 $\mathrm{U}$ and abciximab (ReoPro) $13 \mathrm{mg}$.

Significant stenosis $(66 \%)$ was seen due to the protruded coil mesh in 1 patient after the procedure (case 8 ). The bailout stent had failed in this patient; however, the patient stayed symptom-free with dual antiplatelet medication and heparinization for 3 days. Remarkably, improvement of the stenosis caused by the protruded coils was noted on follow-up $(45 \%)$ of this and a few other patients (cases 2, 3, 5), with partial natural molding of the protruded loop/mesh into the sac probably due to the long-term exposure to the systemic blood flow.

Delayed in-stent stenosis may also be an important complication of the intracranial stent. However, the risk may be lower for the bailout stents during aneurysmal coiling compared with the stent-assisted angioplasty in atherosclerotic stenosis. According to Fiorella et al, ${ }^{20}$ moderate or severe delayed in-stent stenosis was identified in $5.8 \%$ of stent-remodeled coil embolizations. In our series, suspicious significant (53\%) in-stent stenosis was seen on the 5-month follow-up MRA in 1 patient. Due to the asymptomatic nature of the lesion with good preservation of distal flow, the patient was followed clinically for 7 months without development of any symptoms.

\section{Conclusions}

Bailout self-expandable stent deployment may be a feasible and effective method for relief and prevention of parent artery compromise or coil migration caused by prolapsed or unstable coil loops during embolization of aneurysms. This method may be limited in effect when molding small-diameter coil loops in small aneurysms. Long-term follow-up of the delayed effects of these stents and the stabilized loops may be necessary.

\section{References}

1. Fessler RD, Ringer AJ, Qureshi AI, et al. Intracranial stent placement to trap an extruded coil during endovascular aneurysm treatment: technical note. $\mathrm{Neu}$ rosurgery 2000;46:248-51, discussion 251-53

2. Soeda A, Sakai N, Sakai H, et al. Thromboembolic events associated with Guglielmi detachable coil embolization of asymptomatic cerebral aneurysms: evaluation of 66 consecutive cases with use of diffusion-weighted MR imaging. AJNR Am J Neuroradiol 2003;24:127-32

3. Vinuela F, Duckwiler G, Mawad M. Guglielmi detachable coil embolization of acute intracranial aneurysm: perioperative anatomical and clinical outcome in 403 patients. J Neurosurg 1997;86:475-82

4. Cronqvist M, Pierot L, Boulin A, et al. Local intraarterial fibrinolysis of thromboemboli occurring during endovascular treatment of intracerebral aneurysm: a comparison of anatomic results and clinical outcome. AJNR Am J Neuroradiol 1998;19:157-65

5. Benitez RP, Silva MT, Klem J, et al. Endovascular occlusion of wide-necked aneurysms with a new intracranial microstent (Neuroform) and detachable coils. Neurosurgery 2004;54:1359-67, discussion 1368

6. Brooks NP, Turk AS, Niemann DB, et al. Frequency of thromboembolic events associated with endovascular aneurysm treatment: retrospective case series. J Neurosurg 2008;108:1095-100

7. Derdeyn CP, Cross DT 3rd, Moran CJ, et al. Postprocedure ischemic events after treatment of intracranial aneurysms with Guglielmi detachable coils. J Neurosurg 2002;96:837-43

8. Cekirge HS, Saatci I, Ozturk MH, et al. Late angiographic and clinical follow-up results of 100 consecutive aneurysms treated with Onyx reconstruction: largest single-center experience. Neuroradiology 2006;48: $113-26$

9. Dinc H, Kuzeyli K, Kosucu P, et al. Retrieval of prolapsed coils during endovascular treatment of cerebral aneurysms. Neuroradiology 2006;48:269-72

10. Moret J, Cognard C, Weill A, et al. Reconstruction technic in the treatment of wide-neck intracranial aneurysms: long-term angiographic and clinical results—apropos of 56 cases [in French]. J Neuroradiol 1997;24:30-44

11. Sugiu K, Martin JB, Jean B, et al. Rescue balloon procedure for an emergency situation during coil embolization for cerebral aneurysms: technical note. J Neurosurg 2002;96:373-76

12. Biondi A, Janardhan V, Katz JM, et al. Neuroform stent-assisted coil embolization of wide-neck intracranial aneurysms: strategies in stent deployment and midterm follow-up. Neurosurgery 2007;61:460-68, discussion 468-69

13. Wanke I, Doerfler A, Schoch B, et al. Treatment of wide-necked intracranial aneurysms with a self-expanding stent system: initial clinical experience. AJNR Am J Neuroradiol 2003;24:1192-99

14. Fiorella D, Albuquerque FC, Deshmukh VR, et al. Usefulness of the Neuroform stent for the treatment of cerebral aneurysms: results at initial (3-6-mo) follow-up. Neurosurgery 2005;56:1191-201, discussion 1201-02

15. Broadbent LP, Moran CJ, Cross DT 3rd, et al. Management of Neuroform stent dislodgement and misplacement. AJNR Am J Neuroradiol 2003;24:1819-22

16. Ebrahimi N, Claus B, Lee CY, et al. Stent conformity in curved vascular models with simulated aneurysm necks using flat-panel CT: an in vitro study. AJNR Am J Neuroradiol 2007;28:823-29

17. Fiorella D, Albuquerque FC, Han P, et al. Strategies for the management of intraprocedural thromboembolic complications with abciximab (ReoPro). Neurosurgery 2004;54:1089-97, discussion 1097-98

18. Workman MJ, Cloft HJ, Tong FC, et al. Thrombus formation at the neck of cerebral aneurysms during treatment with Guglielmi detachable coils. AJNR Am J Neuroradiol 2002;23:1568-76

19. Cloft HJ, Samuels OB, Tong FC, et al. Use of abciximab for mediation of thromboembolic complications of endovascular therapy. AJNR Am J Neuroradiol 2001;22:1764-67

20. Fiorella D, Albuquerque FC, Woo H, et al. Neuroform in-stent stenosis: incidence, natural history, and treatment strategies. Neurosurgery 2006;59:34-42 